\title{
On-site calibration-based estimation method of forward seabed elevation using forward scan sonar
}

\author{
Juhyun Pyo, Member, IEEE, Hyeonwoo Cho, Member, IEEE, and Son-Cheol Yu, Member, IEEE
}

\begin{abstract}
This paper addresses a novel calibration based estimation method to measure the forward seabed elevation (FSE) where an area illuminated by forward scan sonar (FSS) for unmanned underwater vehicles (UUVs).The conventional method measures the FSE using one or two down-facing single-beam sonars; this method can, however, cause errors. In order to accurately measure the FSE, a sensor capable of measuring the two-dimensional space of the seabed is needed, such as FSS. The method proposed in this work consists in estimating the FSE by analyzing the acoustic beam distribution characteristics of FSS. A novel FSS beam distribution model is proposed, which simplifies beam distribution characteristics. Then, on-site calibration is conducted to estimate the parameters of this model. Based on the estimated model, templates can be generated and compared with FSS data acquired at the experiment in order to estimate geometrical information at the FSE. The FSE of the best matched template is the estimated FSE of the FSS data acquired in the experiment. In order to verify the performance of the proposed method, experiments using an UUV were carried out in an indoor water tank. We also verified that the proposed method performed well even when there were objects on the seabed.
\end{abstract}

Index Terms-On-site calibration, Modeling of forward scan sonar, Localization of UUV, Forward seabed elevation.

\section{INTRODUCTION}

$\mathbf{R}$ ECENTLY, there has been demand for various underwater tasks, such as oceanic rare material investigation, military missions, and environmental surveys [1], [2]. Despite the risky underwater environments, unmanned underwater vehicles (UUVs) have successfully automated these tasks [3]. UUVs also enable us to perform more precise tasks, such as the safety inspection of nearshore and offshore structures [4]. These kinds of sophisticated underwater tasks require environment maps, such as 3D seabed maps, for task planning and localization of UUVs [5], [6]. Furthermore, accurate seabed elevation measurements are the most critical, because most underwater tasks are conducted near the bottom surface, and the seabed elevation is the origin for all tasks. Various methods, utilizing various sensors, are being studied for seabed mapping. Basically, 3D reconstruction based on optical images, which is actively used on land, can be applied first. However, the available sensing methods are restricted in underwater environments. Particularly for the case of underwater optical sensing, lighting should be used in areas where sunlight does not reach. If the turbidity is high, the distance to obtain the optical image is limited, and the UUV must be close to the seabed for acquiring the image. Therefore, forward scan sonars (FSSs) are widely

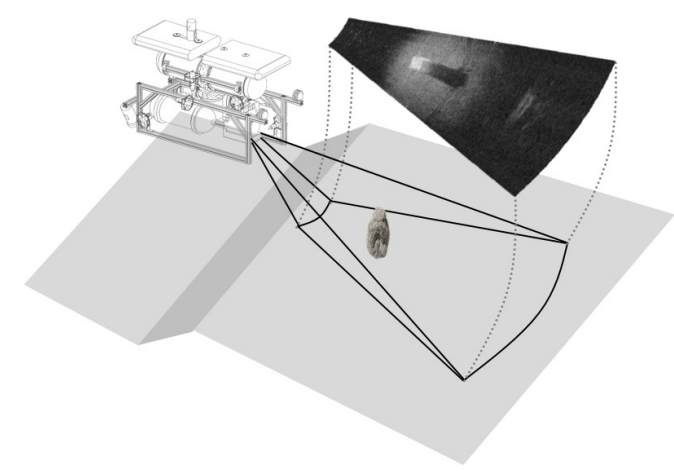

Fig. 1. Forward seabed elevation estimation method using FSS.

used, because vision-based sensing methods have difficulties in reliably performing measurements underwater. In particular, acoustic lens-based FSSs provide high quality acoustic images at a rapid frame rate. Specifications for operating frequency, acoustic beam width, frame rate, and internal beamforming technology vary according to model and manufacturer, but the operating principle of all FSSs is the same. Various methods of 3D reconstruction using FSS have been studied, and there is a study on the stereovision method based on the technology of optical image processing [7]. This method can reconstruct 3D images based on pixel correspondence between two sonar images obtained from different locations. Another method is to use the silhouettes of the shadows in a sonar image [8]. Sonar images can be divided into three regions, namely, shadow, highlight, and background. Of these regions, the shadow region is an area where the acoustic beam is blocked due to the object, and the elevation of these objects can be reconstructed based on the shadow length [8], [9]. These methods are applicable only when there are remarkable objects in the sonar image [10]. However, in the case of general surveys, 3D reconstruction must be performed continuously irrespective of the presence of objects in the sonar image.

Cho et al. propose a continuous 3D reconstruction method using the geometry of FSSs and mobility of the UUV [10]. The proposed method is developed for UUV-based object detection on the seafloor. Based on UUV movement in global coordinates, line scan data obtained from continuous sonar images form 3D point cloud data. When using this 3D point cloud-based mapping method, the seabed elevation of the forward region, where the FSS illuminates, is very important. The authors analyzed the elevation error of the generated object according to the measurement error, and the generated 
map was very sensitive to elevation information.

UUVs with FSS emit acoustic beams diagonally downward to collect data, as shown in Fig. 1. The acoustic beams of FSS are shot in bursts almost simultaneously, and the system receives the returning echo sounds. The emitted beams are fan-shaped, and the received sounds are simply mapped into an acoustic image screen according to the distance. Due to the acoustic nature of FSS, 3D information is compressed to a 2D image screen, and the elevation information in the captured geometry is lost [11]. The FSS has limitations in underwater sensing due to acoustic nature and its display mechanism. For example, it could estimate only 2D information data while the elevation measurement requires $3 \mathrm{D}$ data in underwater. Because of this characteristic of FSSs, it is difficult to estimate the forward seabed elevation (FSE) using FSS [12]. The details are discussed in Section II-B.

Usually, most UUVs acquire altitude information from the bottom surface directly by using Doppler velocity log (DVL) equipment. This combination enables to estimate the FSE of the FSS. The 3D reconstruction method proposed by Cho et al., is based on the assumption that the FSE and the measured downward altitude from the DVL are the same [10]. However, if the measured altitude of the UUV differs from the actual FSE, as shown in Fig. 1, a large position error occurs. If the seafloor is sufficiently flat, this assumption will work well, but it will cause a very fatal error in areas with sloping bottoms or lots of objects. Another conventional method to measure the FSE is to use additional single-beam down-facing sonar (SDS). In case of the seafloor is mostly flat plane surface, this single point or spot based measurement is valid. However, if the seafloor has difference condition, it might cause problem. Moreover, additional SDS means additional cost, and there is a difficulty in matching the point of SDS with the point of the FSS where each beam touches the bottom. In order to complement the lack of elevation information in the FSS data, using additional sensor such as DVL or SDS is a very costly method. Due to the characteristics of UUVs, which are sensitive to very small changes in structure or appearance, the weight and volume increase of UUV due to additional sensors is a kind of risk to its operation. Despite these desperate measures described above, it is still difficult to trust the FSE that have been supplemented.

In this paper, we propose a novel method to estimate FSE by using only FSS without additional sensor. The detailed description of the proposed FSE estimation method is given in Section IV, and the specific performance of the proposed method is verified through experiments in Section V.

\section{BACKGROUND OF FSS}

\section{A. Geometry of FSS}

FSS is a system that acquires echo sounds in parallel by sending multi-acoustic beams in many directions at the same time. Figure 2 shows the configuration of multi-beam in FSS and the set coordinate system of the FSS. Each beam of the FSS has a very narrow horizontal angle, but its vertical angle is relatively wide [9]. These multiple beams together are represented as the field of view in the horizontal direction.

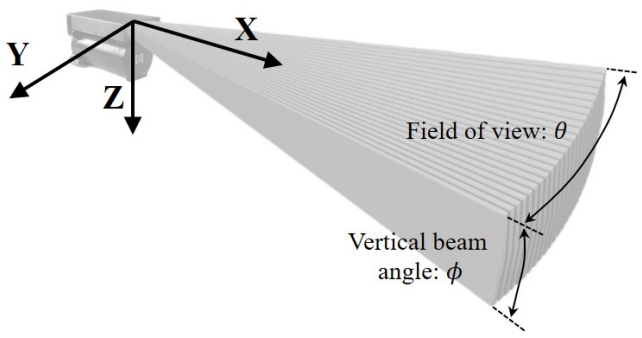

Fig. 2. Acoustic beam configuration in FSS.

(a)

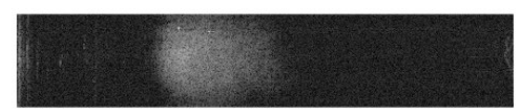

(b)

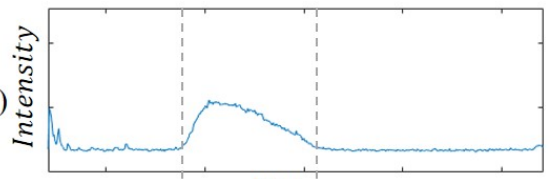

Distance

(c)

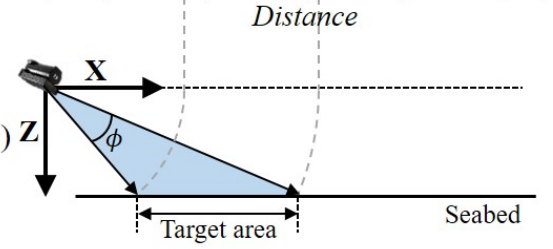

Fig. 3. Geometrical relationship of the FSS and its data, (a) FSS image, (b) Distribution of beam intensity, (c) Beam geometry viewing in XZ plane.

While underwater, the UUV scans the forward seabed using FSS for obstacle avoidance and object recognition. At this time, the UUV is generally controlled keeping a certain depth. There is no rolling or yawing in the FSS motion; only the pitch change is used and changed using a tilting device. Because UUVs are stable in the roll and pitch directions [13], it is not necessary to consider this.

The FFS is mainly used to insonify the forward seafloor for object recognition, obstacle avoidance. The image in Fig. 3 (a) is sonar data in which the intensity of the echo that the beam of the sonar injected diagonally is returned from the bottom surface, and expressed by the distance and the horizontal angle. Each horizontal line in this image is data corresponding to each beam of FSS. Figure 3 (b) and (c) show the geometry of the process of generating data at each beam of FSS. Looking at the geometry of a single beam with respect to the $X Z$ plane, the echoes of the scanned beam reflected from the same distance are projected to one point. The distribution of beam intensity, result of scanning the seabed of a single beam is shown in Fig. 3 (b), and shows a gentle hill pattern. This pattern tends to vary in height and slope depending on the distance and angle between FSS and the bottom surface. If we can model this unique beam pattern, we can estimate the distance and angle between the FSS and the bottom surface by comparing the modeling result with the acquired frame.

As shown in the Fig. 4 (a), raw FSS data is defined as a FSS frame in the form of a two-dimensional array. Figure 4 (b) 

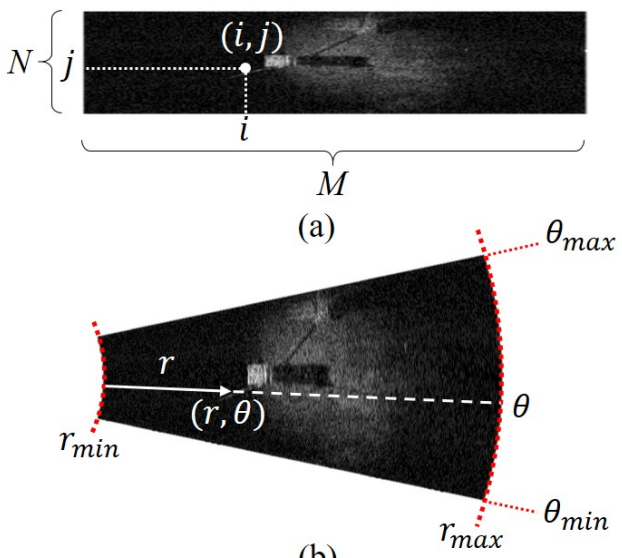

(b)

Fig. 4. Representations of FSS data, (a) Raw FSS data, (b) Polar coordinate type image.

is a processed FSS image as polar-coordinate form from FSS frame to explicitly show the beam configuration. FSS frame is a two-dimensional matrix of $M$ by $N$ for $1 \leq i \leq M$ and $1 \leq j \leq N$, and each pixel value $I(i, j)$ has a gray scale value from 0 to 255 . Row $j$ and column $i$ correspond to the azimuth angle of the individual beams among the field of view and the distance from the FSS, respectively.

The intensity distribution of the $j$ - $t h$ beam can be expressed as $I_{j}(i)$. To analyze the tendency of the whole beam, the mean intensity distribution $\bar{I}(r)$ is mainly use, which can be derived as

$$
\bar{I}(i)=\sum_{j=1}^{N} \frac{I_{j}(i)}{N} .
$$

According to FSS geometry, each pixel index $(i, j)$ can be assigned to polar coordinate $(r, \theta)$. The minimum and maximum distance of the measurable FSS frame are defined as $r_{\min }$ and $r_{\max }$, and these values are selected according to the area to be scanned, among several set values provided by the FSS maker by default. The column index $i$ in the frame can be converted to a distance $r$ using the following equation:

$$
r=r_{\min }+\left(\frac{r_{\max }-r_{\min }}{M-1}\right) \cdot(i-1) .
$$

Using Eq. 2, the mean intensity $\bar{I}(i)$ can be expressed as $\bar{I}(r)$, and a set of $\bar{I}(r)$ for all distance in each frame could be defined as the frame profile, $\mathbb{I}=$ $\left\{\bar{I}\left(r_{\min }\right), \cdots, \bar{I}(r), \cdots, \bar{I}\left(r_{\max }\right)\right\}$.

The FSS frame, in Fig. 4 (a) shows data of $N$ beams in a two-dimensional array. The pixel $(i, j)$ is a value measured by the receiver at the $j$-th beam in terms of the resolution of $I_{j}(i)$. Each pixel value can be represented as a vector, and the $j$-th beam can be assumed as a set of vectors [14], [15].

Figure 5 (a) shows the FSS frame range of the acoustic beam. Section of $j$-th beam shows the formation of the emitted beam corresponding to the FSS frame range $\left(r_{\min }<r<\right.$ $\left.r_{\max }\right)$. A continuous beam can be represented as set of the several vectors, where each vector $\hat{R}$ has a magnitude $r$ and

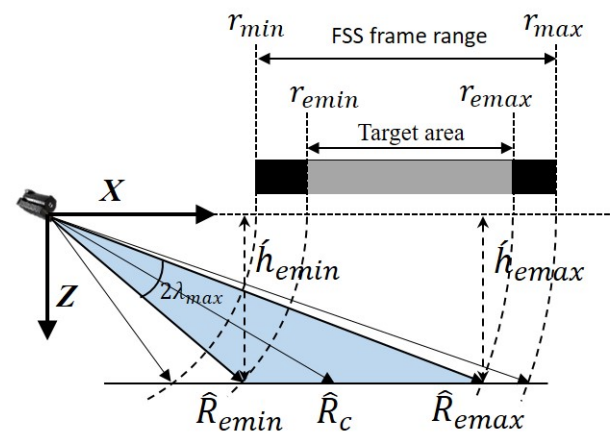

(a)

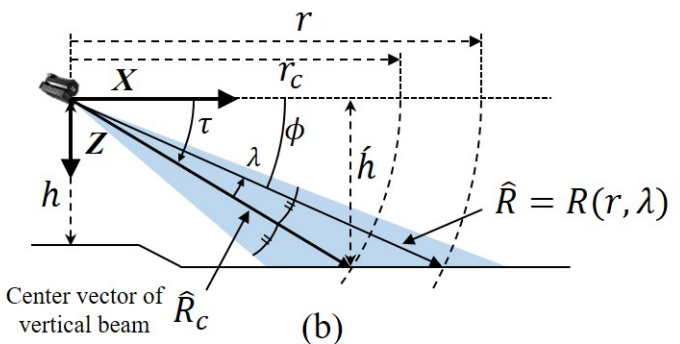

Fig. 5. Side views for geometry of FSS. (a) Frame range of section beam, (b) Vectorization of section beam.

an angle $\lambda ; \hat{R}=r \angle \lambda$. Among the vectors constituting the vertical beam, a vector located at the center of the beam is defined as a center vector, $\hat{R}_{c}$. The vector received at the nearest distance, $r_{\text {emin }}$ from the echo signal for the target area in the frame is defined as $\hat{R}_{\text {emin }}$, and the vector received at the farthest distance, $r_{\text {emax }}$ is defined as $\hat{R}_{\text {emax }}$. In Fig. 5 (b), the tilt angle $\tau$ and the vertical angle $\lambda$ are defined based on the center vector, $\hat{R}_{c}$. Since the FSS data includes only the distance, $r$ and horizontal angle, $\theta$ without the elevation angle $\phi$, it should be estimated using FSS geometry. Knowing the accurate altitude $\hat{h}$ of the bottom surface, the elevation angle $\phi$ can be estimated, and the angle $\lambda$ of each vector can also be estimated as follows:

$$
\lambda=\tau-\phi=\tau-\arcsin \left(\frac{\grave{h}}{r}\right) .
$$

Assuming that the seafloor is flat, the $\tau$ is the tilt angle fixed by setting value of FSS, and FSS beam distribution can be modeled by estimating only the $\lambda$.

\section{B. Difficulties and contributions}

The sonar image expresses the received reflected echo as a value for distance. Due to the nature of this FSS, if the objects are at the same range in the same beam but at different elevation, they could not sort them out [9]. Due to the nature of this FSS, if the objects are at the same range in the same beam but at different elevation, they could not sort them out [9]. For example, as shown in Fig. 6, if objects $O_{A}, O_{B}$ and $O_{C}$ having different heights are located at the same range $\left(r_{A}=r_{B}=r_{C}\right)$ from the FSS, they are matched to the same pixel with distance, $r$ in FSS frame. Since this frame contains only information about the horizontal angle and the distance 


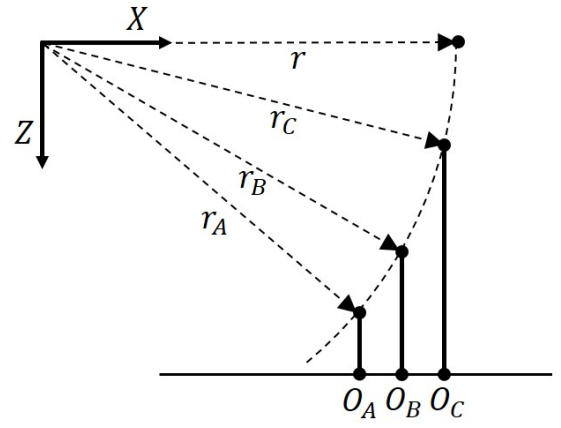

Fig. 6. Ambiguity of elevation angle.

but no elevation angle, it is not possible to determine the 3D position. The position of not only objects but also the seabed cannot be determined.

There are some existing studies to overcome these difficulties, and these studies can be roughly divided into two methods. First, it is a method of using known object such as a landmark (usually preset artificial structures) to calculate the elevation of the target based on the already known geometrical information [16]. Although it is possible to estimate the exact position at various altitudes, there are disadvantages that the position can be estimated only in the limited area where the landmark is installed and it should be installed in advance. The following method assumes that the seabed is sufficiently flat, and then the altitude of the UUV which is measured by using the altimeter (installed to the UUV) is applied as the altitude of the target area (where FSS is looking) [7], [8], [10]. This method is very simple to apply and is quite accurate when the seabed is actually flat. However, it cannot be guaranteed that the seabed will always be flat, and a large error occurs even at a slight slope. These methods can estimate the altitude (or elevation angle) of the target, but the known object or additional sensor other than the FSS is essentially required.

Unlike these methods, we propose a novel method of estimating FSE only within FSS frame without the need for additional factors. The proposed method accumulates experimental data on the acoustic beam distribution pattern of the seabed surface at various angles and distances. Based on these data, we can design a beam distribution model and estimate the FSE by generating multiple templates from this model. The detailed procedure of the proposed method will be described in detail in the next section.

\section{CONCEPT}

The proposed method estimates the FSE only using FSS data. This sensing approach enables UUVs to measure accurate, reliable FSE with small computing power. The beam intensity of the FSS increases when it is close to the center and decreases as the distance increases. Based on these beam characteristics, an independent beam distribution pattern is generated that reflects on the bottom surface depending on the distance and angle of the FSS. By modeling the beam distribution pattern and comparing the result with the beam pattern of the actually obtained FSS, the distance and angle between the FSS and the bottom surface can be estimated. A beam distribution model is required to generate various beam distribution patterns for FSS angles and distances.

In order to estimate the parameters of the beam distribution model, the data acquisition (DA) experiment is conducted. This process of estimating model parameters based field experiments is called on-site calibration. Since this paper aims to estimate the FSE using FSS, it accumulates frames at various distances to the bottom surface in the DA experiment. Based on the accumulated data from the DA experiment, the beam distribution model parameters for the FSE can be estimated. The beam distribution model and the model parameter functions are derived from the sonar equation that be introduced in the next section. As a result of the estimated model, the beam distribution pattern of seabed under any condition can be generated. This beam distribution pattern is called template. Generated templates are compared with the acquired frame in FSE estimation (FE) experiment to estimate the FSE.

\section{A. Sonar equation}

The sonar equation is an important concept in modeling of FSS. It reflects the substantial relationship between media and sonar, sonar systems and detected targets and sonar operating conditions [17]. Once sonar sensor and targets are selected, the main parameters of the sonar equation are confirmed. Sonar performance depends on the ocean environment, such temperature, pressure, depth, and characteristics of the seabed. This effect can vary depending on the time, as well as the environment. Sonar equation is a key concept in the modeling of FSS, because they objectively reflect the main parameter in beam distribution model of FSS.

There are three types of parameters in the sonar equation [17], [18]:

1) Parameters defined by the sonar system: $S L$ (Source level); The measure of the amount of the acoustic energy input by the transmitter. $R I$ (Recognition index); This index refers to the intensity of the measured echo return. This value is recognized only when the minimum output SNR is exceeded.

2) Parameters defined by the ocean environment: $T L$ (Transmission loss); This indicate the propagation attenuation, the combination of spreading loss and absorption loss of a sound wave. $N L$ (Noise level); The magnitude of unwanted spurious signals in the sonar input. This may consist of ambient noise, reverberation or self-noise of a sonar platform.

3) Parameters defined by the target: TS (Target strength); The ability of a target to gather received acoustic energy and re-transmit it to directed back in the direction of the sonar.

All quantities of parameters are expressed in decibels $(d B)$. Assuming that the power of the transmitting signal is $S P$, the source level can be expressed as $S L=S P+D I(d B)$, where $D I$ is the transmitter directional coefficient. If the transmitter is omni-directional, then $D I=0 d B$ [17].

The FSS used in our experiment is an active sonar that builds up each frame measured with eight transmit/receive cycles [9]. Figure 7 shows the schematic diagram in active sonar equation.

The following sonar equation can be used to track all the factors associated with the acoustic echo process [17], [18]. 


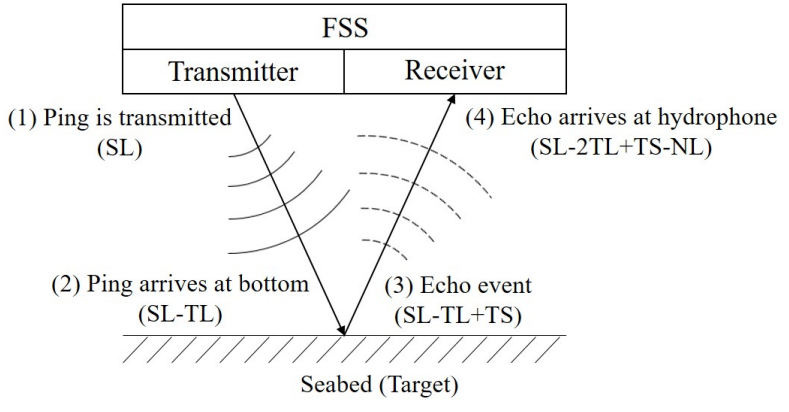

Fig. 7. Derivation of active sonar equation.

$$
R I=S L-2 T L+T S-N L
$$

Looking at the factors of this model, we can determine that the FSS data is affected by the characteristics of the experimental environment (media and target) and the sensitivity of the FSS. Although these factors can be used to design an accurate beam distribution model, it is difficult to quantify the individual parameters in sonar equation considering environments. Therefore, we used the method, in this study, simplified the beam distribution model of FSS as a function of distance and angle using sonar data obtained from the experimental site. The procedure of estimating the beam distribution model of FSS through DA experiment is described in the following section.

\section{B. Modeling}

In order to estimate the parameters of the model for the beam distribution characteristics, frames must be collected. According to the geometry of FSS, when FSS emits a beam diagonally, the elevation angle, $\phi$ of each vector, $\hat{R}$ for this vertical beam cannot be estimated. In order to overcome this uncertainty, the DA experiment is conducted at the configuration of FSS as shown in Fig. 8. The area where the acoustic beam is reflected from the bottom is represented as beam profile data in between $r_{\text {emin }}$ and $r_{\text {emax }}$. Because the FSS is looking at the bottom surface perpendicularly, the intensity value of beam profile is greatest where the center vector of the vertical beam, $\hat{R}$, and the intensity value gradually decreases with increasing distance from the center.

When the tilt angle of the FSS is perpendicular to the bottom surface $\left(\tau=90^{\circ}\right)$, the elevation angle, $\phi$ of the each vector, $\hat{R}$ can be estimated, since the beam profile data and vectors of the vertical beam are matched. The distance, $r_{C}$ of the center vector is equal to the minimum distance, $r_{\text {emin }}$ to the bottom (where the beam profile is maximum), therefore, center vector, $\hat{R}_{C}$ is equal to the vector $\hat{R}_{e m i n}$. At this time, the distance, $r_{\text {emin }}$ is equal to the altitude, $h$ of the FSS. From this altitude, the vertical angle, $\lambda$ for all vectors, $\hat{R}_{C}$ of beam can be calculated using Eq. 3 .

The beam profile in each altitude can be expressed as a beam distribution as shown in Fig. 9. The beam distribution represents the beam intensity, $\bar{I}(\lambda)$ with respect to the calculated vertical angle, $\lambda$. The trend of beam distribution is a

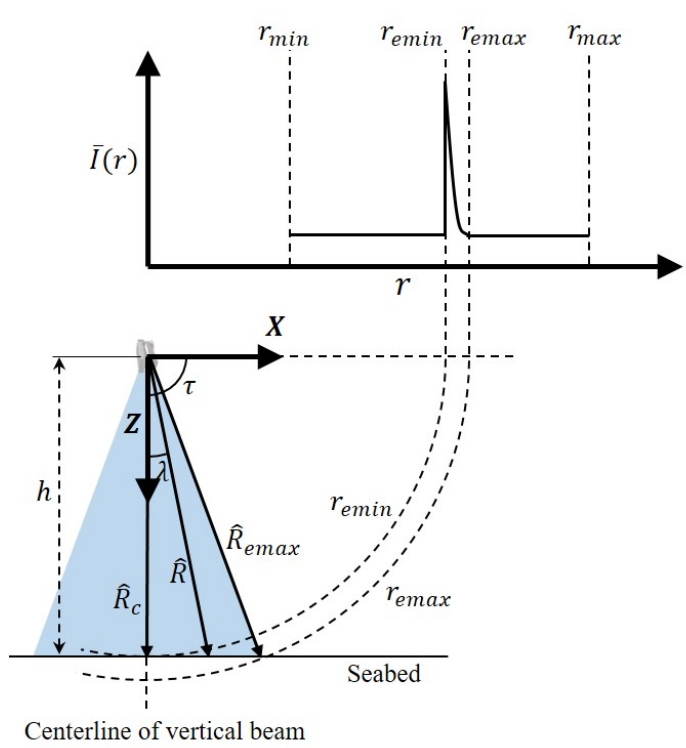

Fig. 8. Geometry for FSS during the DA experiments and beam profile data.

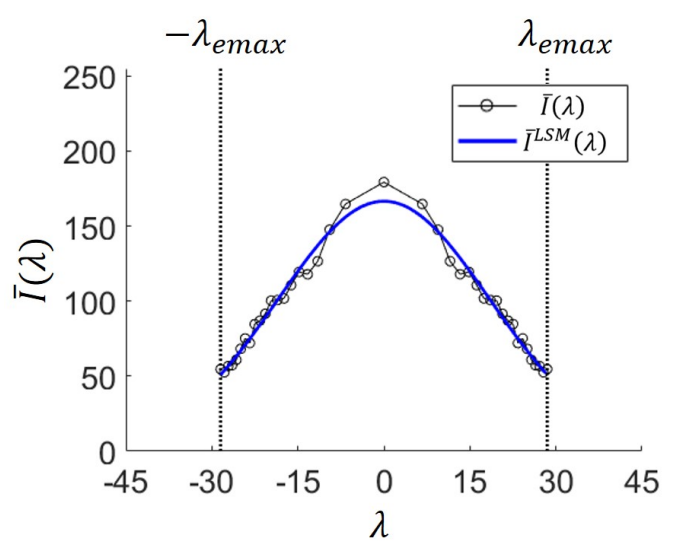

Fig. 9. Beam distribution which is relationship between intensity value $\bar{I}$ and vertical angle $\lambda$, altitude $h$ was $2.5 \mathrm{~m}$.

symmetric bell curve shape in which the beam intensity at the center $(\lambda=0)$ is highest and then decreases as the beam angle increases. During the DA experiment, the data of the beam profile and beam dispersion at each altitude are continuously accumulated.

The beam distribution model that can model the trend of the whole beam profiles during DA experiment is as follows.

$$
\bar{I}=F(\hat{R}) \cdot I_{0}
$$

In this beam distribution model, a single beam is represented by several vector sets, and each vector $\hat{R}$ is modeled as the intensity value $\bar{I}$ of a frame. In this model, $I_{0}$ means the intensity of the emitted sound source, and is the maximum value $\left(I_{0}=255\right)$ that the pixel of the frame can have. $F$ is a function of the amount of signal lost during the movement of the sound source corresponding to each vector, and is modeled based on the sonar equation. Model parameters are inferred from the beam distribution pattern of the frame acquired through the DA experiment. The beam distribution trend for 
the vertical beam angle $\lambda$ can be approximated as a Gaussian distribution (in Eq. 6) [19].

$$
F(\hat{R})=G(r) \cdot e^{-\left(\frac{\lambda^{2}}{2 \cdot H(r)^{2}}\right)}
$$

Because the model parameters are estimated by the data obtained via DA experiment, the beam distribution model includes the characteristics of the experimental environment, such as the medium in which the acoustic beam travels and the seabed, where the echo sound is reflected. The parameter $G(r)$ is the height of the curve's peak, and $H(r)$ is the standard deviation which determines the width of the bell curve.

$$
\begin{aligned}
& G(r)=\alpha_{1} \cdot \alpha_{2}{ }^{(r)}+\alpha_{3} \\
& H(r)=\beta_{1} \cdot \beta_{2}{ }^{(r)}+\beta_{3}
\end{aligned}
$$

The parameters $G(r)$ and $H(r)$ of each frame, obtained according to the changes in altitude $h$ in the DA experiment, are modeled as an exponential function with the distance $r$ as a variable. Therefore, six parameters $\left(\alpha_{1}, \alpha_{2}, \alpha_{3}, \beta_{1}, \beta_{2}\right.$ and $\beta_{3}$ ) are used to determine the final beam distribution model through the DA experiments.

The method for estimating the parameters of the model using the frames obtained via the DA experiment is as follows. First of all, we can assume that the lengths $r$ of all vectors ${ }^{\forall} \hat{R}$ are approximated equal to minimum distance $r_{e m i n}$, because the effective distance between $r_{e m i n}$ and $r_{e \max }$ is much smaller than the altitude $h$ during DA experiment. The minimum distance $r_{\text {emin }}$ is equal to the altitude $h$ at each frame. This assumption can solve the parameter estimation equation much simpler, and can be applied to the beam distribution model as follows:

$$
\bar{I}^{L S M}=F(h \angle \lambda) \cdot I_{0}=G(h) \cdot e^{-\left(\frac{\lambda^{2}}{2 \cdot H(h)^{2}}\right)} \cdot I_{0} .
$$

In order to approximate the beam distribution $\bar{I}^{L S M}$ obtained from the DA experiment with the Gaussian distribution of the above mentioned model, we can use the least square method (LSM) to obtain the functions of parameters $G$ and $H$ for each frame.

\section{PROPOSED METHOD}

In this paper, we propose a novel FSS model, experimentally determine the model parameters, estimate the FSE in FSS data by comparing the generated model-based templates. The proposed method proceeds as follows.

In the section IV-A, a DA experiment is conducted to obtain FSS data including the beam reflection characteristics of the seabed in the experimental environment according to the altitude change. The parameters of beam distribution model of FSS could be calculated via approximations based on the FSS data from DA experiments. In the section IV-B, templates can be generated based on the inferred parameters of beam distribution models. In the section IV-C, The FSE can be estimated by comparing the generated templates with the beam distribution of the FSS data acquired in real time during the FE experiment.

\section{A. Data acquisition experiments}

In this study, a DA experiment is the process of estimating the parameters of the beam distribution model by obtaining FSS data from a field experiment and approximating this data with the model. In our DA experiments, the procedure for obtaining FSS data is as follows. FSS is attached to the bottom of an UUV with a tilting module, which is used to adjust the irradiation angle. The UUV moves as close to the bottom as possible, and then the FSS is rotated in order to face downwards $\left(\tau=90^{\circ}\right)$, as shown in Fig. 8. Then, the UUV ascends vertically to obtain the acoustic beam distribution according to the changes in altitude $h$, and continuously accumulates frames.

Figure 10 shows the beam profiles and distributions acquired during the DA experiment. The altitude $h_{1}, h_{2}$ and $h_{3}$ are the distance between the FSS and the bottom, $1 m, 2 m$ and $3 m$, respectively. As the altitude increases, the area where the acoustic beam was reflected from the bottom surface increases, but the range of the effective vertical angle decreases. If the acoustic beam is transmitted far away, the transmission loss due to attenuation increases and the acoustic intensity decreases. These tendencies are consistent with the trend of sonar equation. The steep area at the beginning of the beam profile is similarly occurred in all frames due to noise caused by micro bubbles in the FSS lens. The trends of the parameters, which are the approximation results of all the frames obtained from the DA experiment are in Fig. 11.

The functions of parameters $G$ and $H$ tend to decrease exponentially as altitude $h$ increases. The parameters $\left(\alpha_{1}, \alpha_{2}\right.$, $\alpha_{3}, \beta_{1}, \beta_{2}$ and $\beta_{3}$ ) are inferred by using the LSM from the previous modeling section. If all parameters are estimated, the acoustic beam distribution for all distances and angles can be expressed based on the proposed model.

Figure 12 (a) shows the beam distribution of the frames obtained through the DA experiment which is conducted within 6 meters when the tilt angle is 90 degrees. Figure 12 (b) shows the beam distribution results of the model estimated from the acquired frames, and the proposed sonar model can well represent the beam distribution characteristics including the environment. This results means that the designed beam distribution model and the estimated parameters are acceptable. Like the tendency of the sonar equation, the effective angle and strength of the acoustic beam intensity tend to decrease as the distance increases, in the both beam distributions.

\section{B. Template generation}

Based on the parameters of the model estimated in the previous section, we can generate templates for the desired distances and angles as a criterion for estimating the FSE. The necessary conditions for generating such templates are the tilt angle $\tau$ of the FSS and the range of candidate the FSEs $h$. The tilt angle is set by the user in advance to adjust the scan area of FSS. The templates to be generated have different beam distributions based on the each FSE. Therefore, it is necessary to set the range of possible FSEs for the templates to be generated. Beam distribution $\bar{I}^{T}(r)$ of 


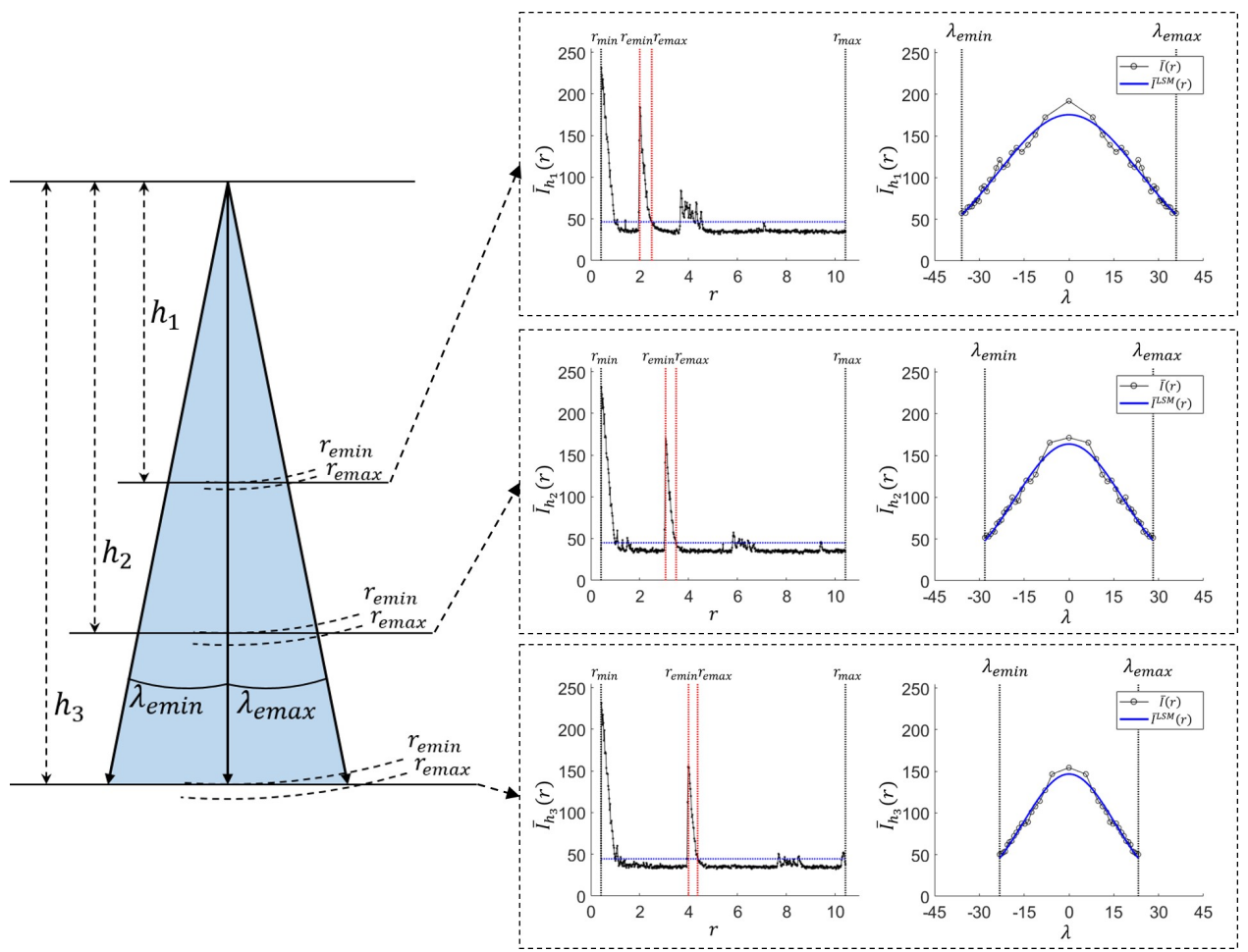

Fig. 10. Example of the beam profiles and distributions for each altitude acquired during a DA experiment.

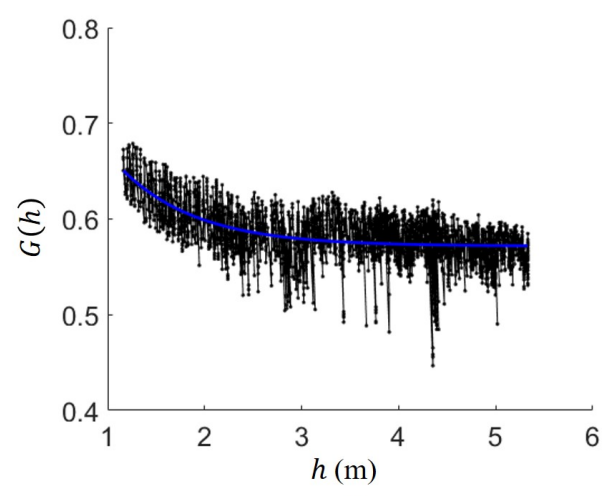

(a)

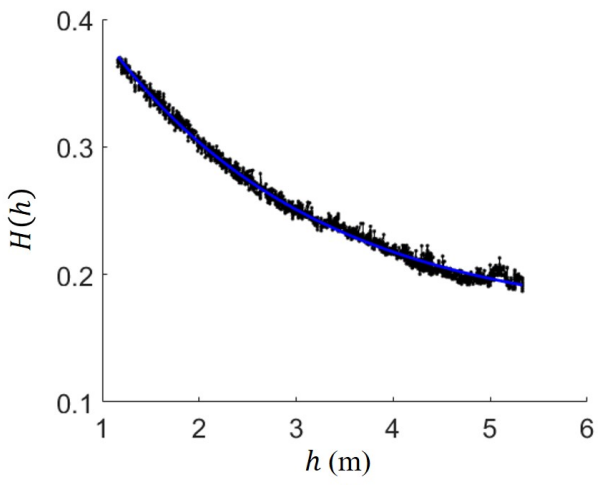

(b)

Fig. 11. The trends of parameters, (a) $G$ and (b) $H$, which are the approximation results of all the frames obtained from the DA experiment

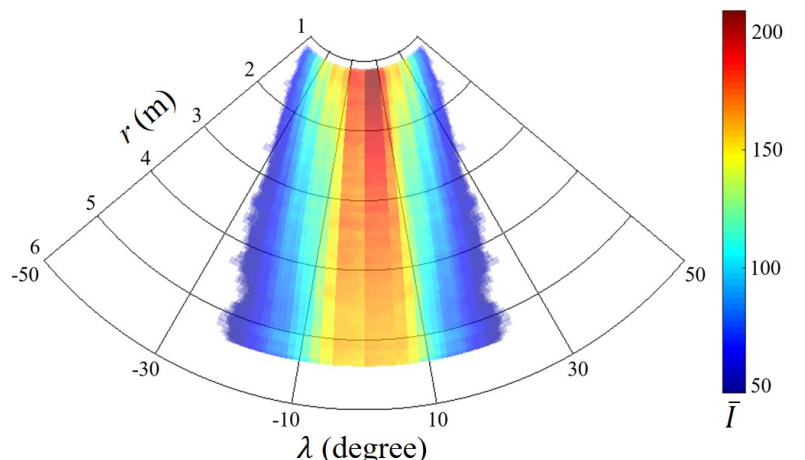

(a)

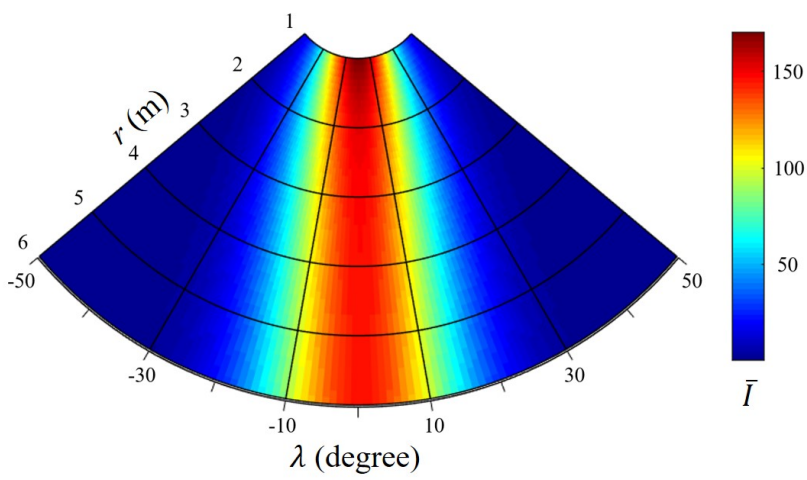

(b)

Fig. 12. Comparison of beam distributions from DA experiment and model estimation, (a) Beam distribution of the obtained frames, (b) Result of the estimated beam distribution model. 


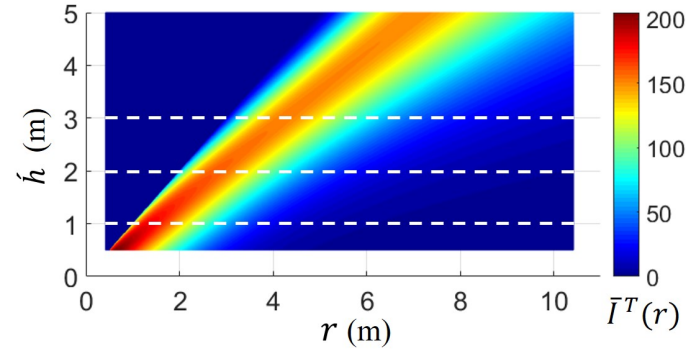

(a)

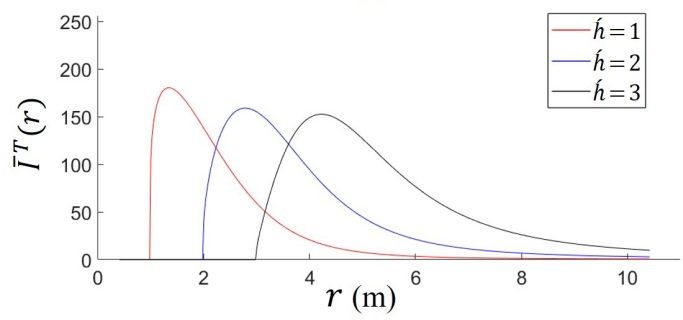

(b)

Fig. 13. Example of a template, (a) Template for a tilt angle $\lambda$ of $45^{\circ}$, and the FSE $h$ ranging from 0.5 to $5 m$, (b) Beam profiles of selected elevations.

template can be calculated by substituting these two conditions as parameters to equation, and the superscript $T$ denotes the beam distribution of the generated template.

$$
\bar{I}^{T}(r)=\left\{\begin{array}{cc}
0, & \text { if } r \leq \hat{h} \\
F(\hat{R}) \cdot I_{0}, & \text { if } r>\hat{h}
\end{array}\right.
$$

If the distance $r$ of the beam vector is less than the FSE $h$, the beam intensity $\bar{I}^{T}(r)$ is assumed to be zero. A template for each candidate FSE can be expressed as: $\mathbb{I}^{T}=\left\{\bar{I}^{T}\left(r_{\min }\right), \cdots, \bar{I}^{T}(r), \cdots, \bar{I}^{T}\left(r_{\max }\right)\right\}$. The templates $\mathbb{I}^{T}$ for $45^{\circ}$ tilt angle and from 0.5 to $5 m$ of FSE $h$ is shown in Fig. 13.

\section{FSE estimation}

The FSE can be estimated by comparing the templates with the FSS frames acquired by the FE experiment. The beam profile, which is the raw frame acquired through the FE experiment, is marked with a superscript $R$. Noise caused by the interference of the transducer and receiver of the FSS is removed from the raw frame. From the noise-canceled raw data, the intensities $\bar{I}^{R}(r)$ larger than the mean intensity, Mean $\left(\bar{I}^{R}(r)\right)$, are set as the beam profile $\mathbb{I}^{M}$ of the FE experiment to be compared with templates: $\mathbb{I}^{M}=$ $\left\{\bar{I}^{M}\left(r_{\min }\right), \cdots, \bar{I}^{M}(r), \cdots, \bar{I}^{M}\left(r_{\max }\right)\right\}$. The raw frame, $\mathbb{I}^{R}$ and processed beam profile, $\mathbb{I}^{M}$ from the FE experiment are shown in the Fig. 11 (a). In the FE experiment, the FSS frame acquired in real time is converted to $\mathbb{I}^{M}$, and this profile is compared with the generated template $\mathbb{I}^{T}$ to calculate the correlation coefficient $\rho_{T M}$.

$$
\rho_{T M}=\operatorname{correl}\left(\mathbb{I}^{T}, \mathbb{I}^{M}\right)=\frac{\operatorname{cov}\left(\mathbb{I}^{T}, \mathbb{I}^{M}\right)}{\operatorname{std}\left(\mathbb{I}^{T}\right) \operatorname{std}\left(\mathbb{I}^{M}\right)}
$$

In this matching process, the value at the point that yields the highest correlation coefficient $\rho_{T M}$ is determined as the

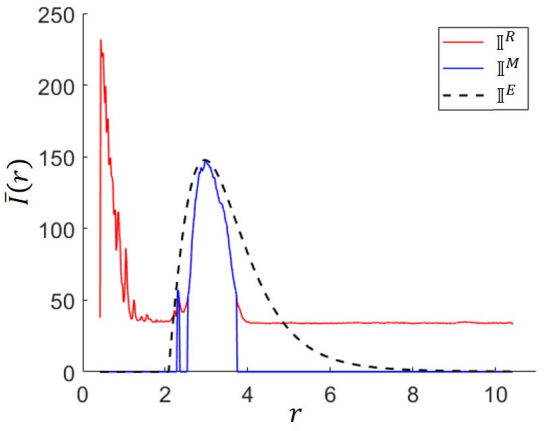

(a)

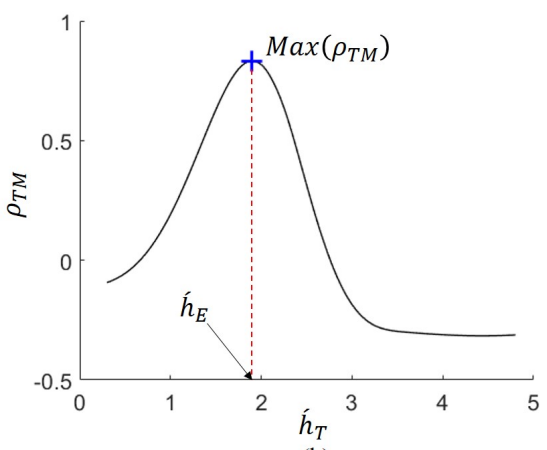

(b)

Fig. 14. (a) Results when calculating the correlation coefficient value. (b) Comparison of the results of the beam profile obtained in FE experiment and with template.

FSE of each frame. Figure 14 (b) shows our results when calculating the correlation coefficient values for the templates of the candidate FSEs. When the correlation coefficient value $\rho_{T M}$ is highest, the template FSE $h_{T}$ is applied as estimated FSE $h_{E}$, and the template $\mathbb{I}^{T}$ is then defined as the best matching profile $\mathbb{I}^{E}$. The superscript $E$ is defined to be used for the best estimated result. Figure 14 (a) represents a comparison of the $\mathbb{I}^{M}$ obtained from the FE experiment and the $\mathbb{I}^{E}$ of the best matching template.

\section{EXPERIMENTS}

\section{A. Experiment environment}

The experiments to verify the proposed method are as follows: 1) the DA experiment is performed to obtain the parameters of the beam distribution model and make the templates, and 2) the FE experiment compares the acquired FSS frames with the generated templates to estimate the FSE.

A hovering type UUV is used for the experiment [13], FSS and various sensors are mounted on the front part of the UUV, as shown in Fig. 15 (a) and (b). The FSS frames were acquired by adjusting the angle of the FSS with a tilting module. The experiments were carried out in an indoor water tank, as shown in Fig. 12 (c), and the bottom surface of the tank was a concrete structure. The experimental environment is set up as shown in Fig. 12 (d).

The specifications of the FSS used in the experiment are shown in Table I. 


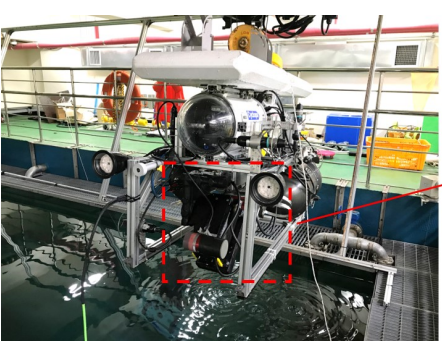

(a)

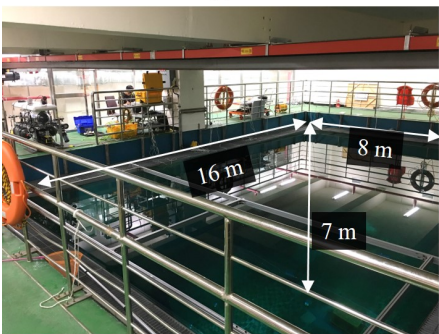

(c)

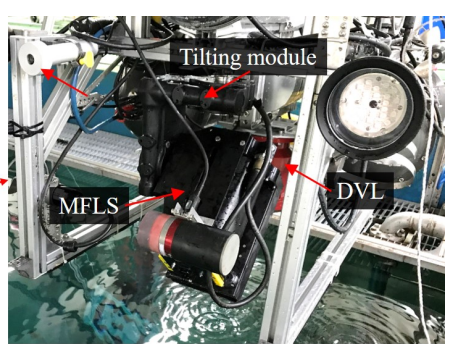

(b)

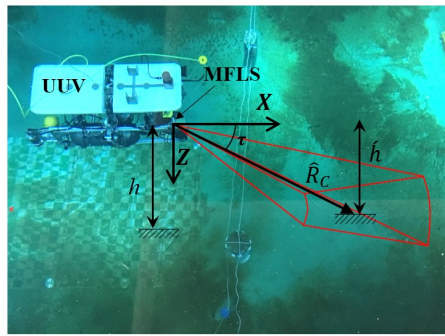

(d)
Fig. 15. Experimental overviews, (a) Hovering type UUV 'Cyclops', (b) Layout of the FSS and front sensors, (c) Indoor water tank, (d) Experiment setup.

TABLE I

SPECIFICATIONS OF THE FSS (DUAL-FREQUENCY IDENTIFICATION SONAR: DIDSON).

\begin{tabular}{cc}
\hline \hline Operating frequency $(\mathrm{MHz})$ & $\mathbf{1 . 1 / 1 . 8}$ \\
\hline Number of beams & $\mathbf{9 6}$ \\
\hline Range $(\mathrm{m})$ & $\mathbf{0 . 4} \sim \mathbf{4 0}$ \\
\hline Field of view $(\mathrm{deg})$ & $\mathbf{2 9}$ \\
\hline Beam width $(\mathrm{deg})$ & $\mathbf{0 . 4} \mathbf{1 4}$ \\
\hline Max. update rate $(\mathrm{Hz})$ & $\mathbf{2 1}$ \\
\hline Max. range resolution $(\mathrm{m})$ & $\mathbf{0 . 0 0 2 5}$ \\
\hline Angular resolution $(\mathrm{deg})$ & $\mathbf{0 . 3}$ \\
\hline Sonar weight $(\mathrm{kg})$ & $\mathbf{7 . 7}$ \\
\hline Dimensions $(\mathrm{m})$ & $\mathbf{0 . 3} \times \mathbf{0 . 2} \times \mathbf{0 . 1 7}$ \\
\hline \hline
\end{tabular}

\section{B. DA experiment}

In the DA experiment, the UUV moves starting from vertical distance of $1 \mathrm{~m}$ from the bottom of tank, rotating the FSS $90^{\circ}$, and ascending to an altitude of $5.5 \mathrm{~m}$ while successively acquiring FSS data. When the UUV is close to the bottom surface, the sonar beam is reflected at the highest intensity and decreases steadily with increasing altitude. The UUV moves based on the altitude value estimated by DVL equipment during the DA experiment. The UUV's altitude is gradually increased and approximately 14000 FSS frames are obtained during DA experiment. The acquired FSS frames can be represented as a single beam distribution. The parameters of the beam distribution model are calculated based on the FSS frames during the DA experiment. From this model, the templates for the desired distances and orientations can be generated, as shown in Fig. 16. Each template shows the tendency of beam distribution versus the FSE for tilt angles $\tau$ of $30^{\circ}, 45^{\circ}$ and $60^{\circ}$.

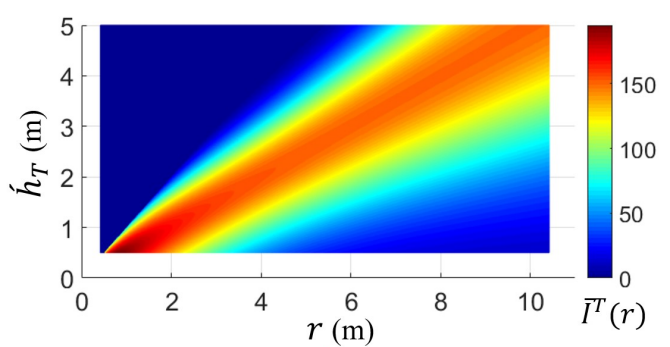

(a)

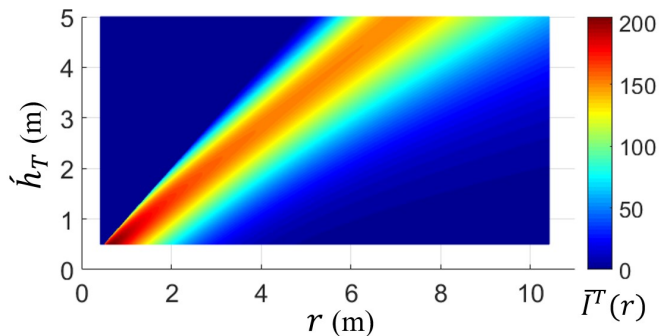

(b)

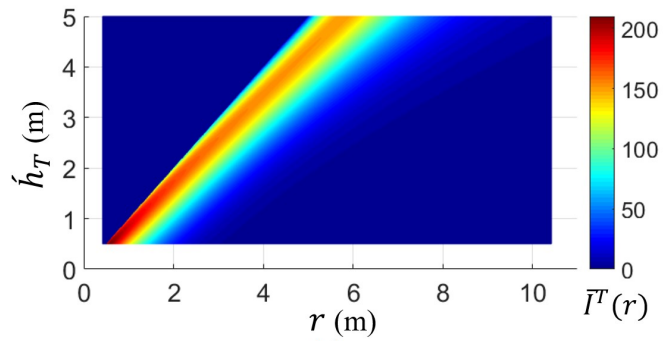

(c)

Fig. 16. Templates of beam profiles for tilt angles of (a) $30^{\circ}$, (b) $45^{\circ}$, and (c) $60^{\circ}$

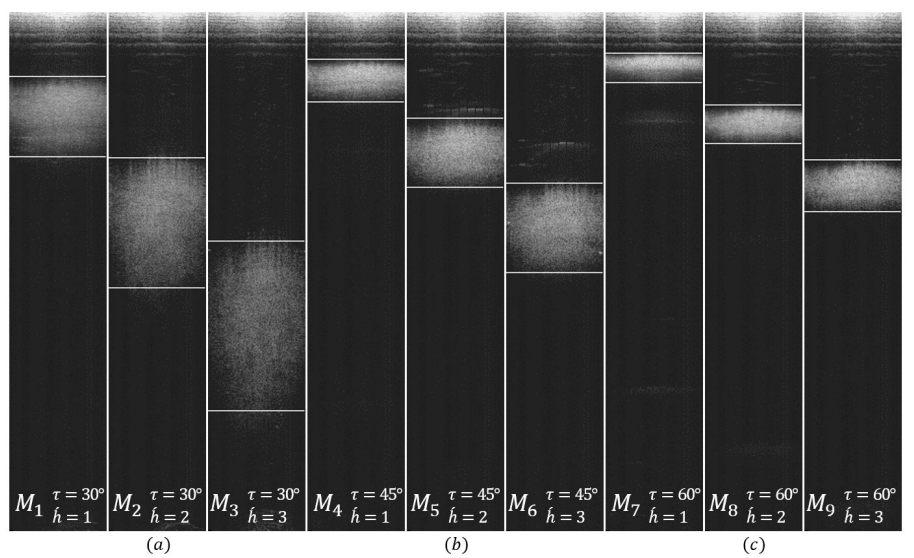

Fig. 17. FSS frames (image of raw FSS data) for tilt angles of (a) $30^{\circ}$, (b) $45^{\circ}$, and (c) $60^{\circ}$.

\section{FE experiment}

In order to verify the accuracy of the generated templates, the FE experiment was conducted for nine combinations of the FSE and tilt angles $\left(1,2\right.$, and $3 \mathrm{~m}$ for tilt angles of $30^{\circ}$, $45^{\circ}$ and $60^{\circ}$ ). Figure 17 shows the acquired frames for each of these conditions.

Table II shows the results of the FSE estimation from the frames obtained at the predetermined positions as shown in Fig. 17. All estimation results are within $10 \%$ error range. 


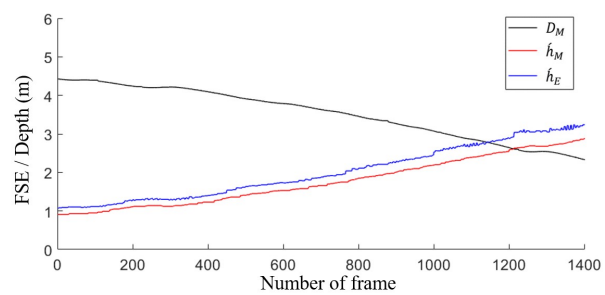

(a)

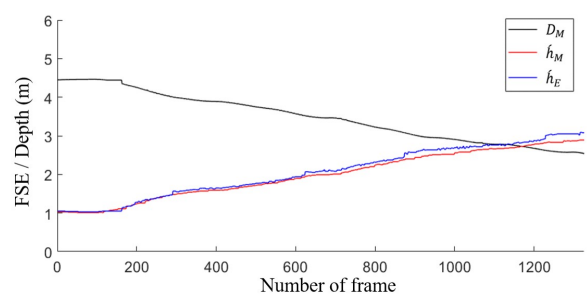

(b)

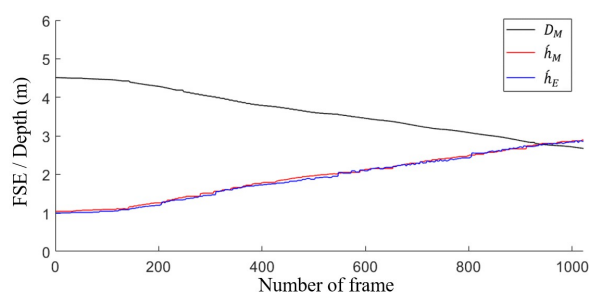

(c)

Fig. 18. Results of FSE estimation at tilt angles of (a) $30^{\circ}$, (b) $45^{\circ}$, and (c) $60^{\circ}$, during the FE experiment with continuous movement of UUV.

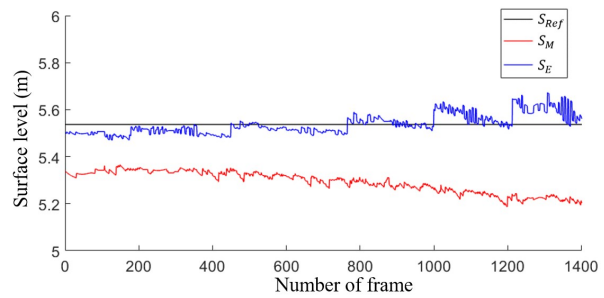

(a)

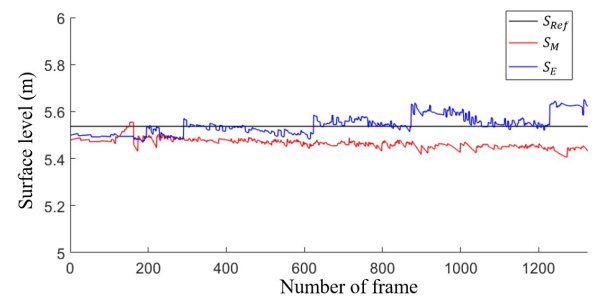

(b)

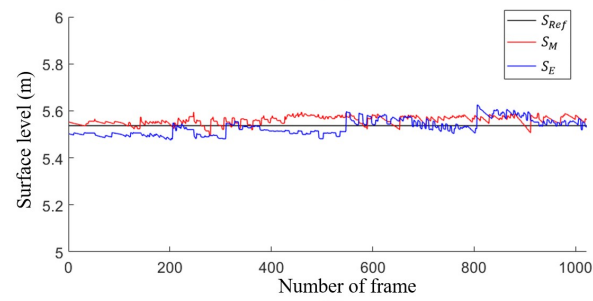

(c)

Fig. 19. Surface level of estimation result for tilt angles of (a) $30^{\circ}$, (b) $45^{\circ}$, and (c) $60^{\circ}$.
TABLE II

RESULTS OF FSE ESTIMATION FROM ACQUIRED FRAMES $(m)$.

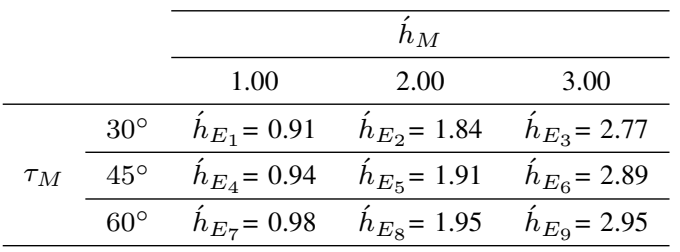

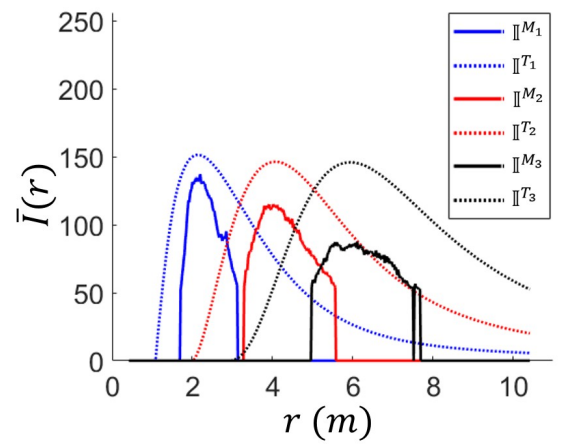

(a)

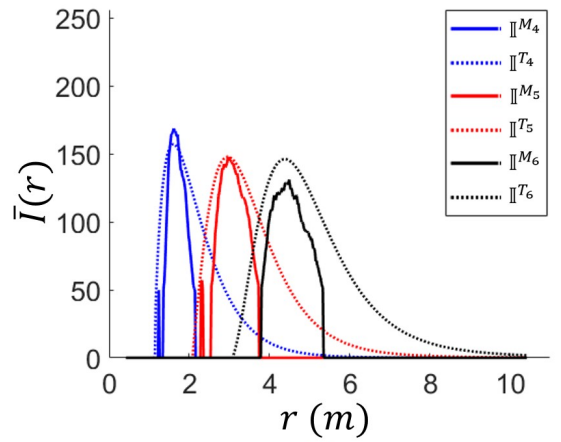

(b)

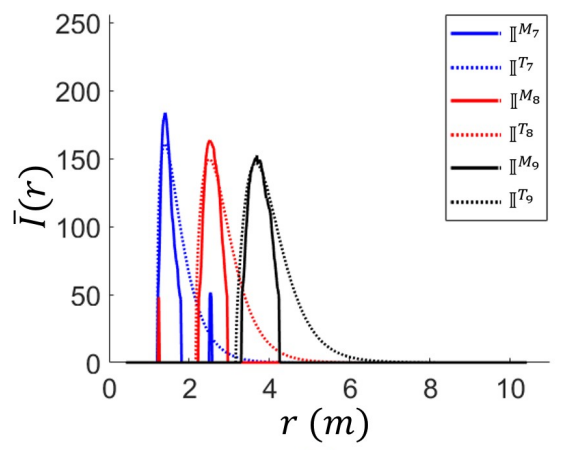

(c)

Fig. 20. Matching results between templates and acquired frames for tilt angles of (a) $30^{\circ}$, (b) $45^{\circ}$, and (c) $60^{\circ}$.

However, the more tilt angle decreases, the more the error of results are increased. This tendency of this error can be more clearly seen in the following experimental results, as shown in Fig. 18. In this experiment, UUV fixes the tilt angle, increases its altitude at the same time, and obtains the frames continuously. The results of this experiment, in 
Fig. 18 represents the UUV's depth, altitude (measured in the experiment), and the FSE estimation for this continuous change of altitude. The other represents of results are in Fig. 19 as a surface level (of water tank) to better understand the estimation accuracy. The surface level is the sum of the elevation information and the depth, and should always be constant. Similar to the previous results, if the tilt angle is large (in Fig. 19 (c)), the surface level, $S_{E}$ is constant and accurate. However, it can be seen that the error continuously occurs at the tilt angle is small. As seen in the results, the measured altitude, $h_{M}$ with DVL also contains some error.

These errors can be understood by the matched template under each condition. Figure 20 shows a beam profile graph of the frames and templates with the highest matching results. As shown in Fig. 20 (c), when the tilt angle was large $\left(60^{\circ}\right)$, the matched templates are very similar to the beam profiles. However, as shown in Fig. 20 (a), the similarity between the matched template and the beam profile decreased if the tilt angle was small $\left(30^{\circ}\right)$. These results occur for the following reasons. In order to infer the parameters of the proposed model, the DA experiment was modeled using only the data measured in the direction perpendicular to the bottom surface, so there is no consideration for the incident angle of the beam on the objects surface. The incident angle with respect to the normal direction of the objects surface considerably affects the actual beam distribution of our model. If the incident angle of the beam is large, the scattering becomes large and the amount of reflection tends to be small. This can be expressed as Lamberts cosine law [20]. In order to compensate for this, frames could be obtained according to changes in the tilt angle of the FSS during the DA experiment and be then applied to our beam distribution model; thus, more accurate templates could be generated.

\section{Comparison method}

In the sonar image, the area where sound waves are returned from the seafloor is called the background. As shown in Fig. 5 (a), the near and far boundaries of the background in sonar image can be expressed as vector $\hat{R}_{\text {emin }}$ and $\hat{R}_{\text {emax }}$, and each vector has the extreme elevation angles of $\lambda_{\max }$ and $-\lambda_{\max }$, respectively. The area from the beam vector $\hat{R}_{\text {emin }}$ to $\hat{R}_{\text {emax }}$ is defined as the vertical beam width and its size is $2 \lambda_{\max }$. The FSEs can be calculated from the elevation angles of the vector $\hat{R}_{\text {emin }}$ and $\hat{R}_{\text {emax }}$ located at the boundary of the background. The elevation angles in both side of the boundary are $\tau+\lambda_{\max }$ and $\tau-\lambda_{\max }$, respectively, and FSE can be obtained by the following equation.

$$
\begin{aligned}
& \hat{h}_{\text {emin }}=r_{\text {emin }}+\sin \left(\tau+\lambda_{\max }\right) \\
& \hat{h}_{\text {emax }}=r_{\text {emax }}+\sin \left(\tau-\lambda_{\max }\right)
\end{aligned}
$$

The tilt angle, $\tau$ can be measured directly by the tilting device.

At this time, if the vertical beam width is constrained, the elevation angle of the background can be inferred [21]. In the specification of FSS used in this paper, the vertical beam width is set to $14^{\circ}$, therefore $\lambda_{\max }$, is $7^{\circ}$. This method is

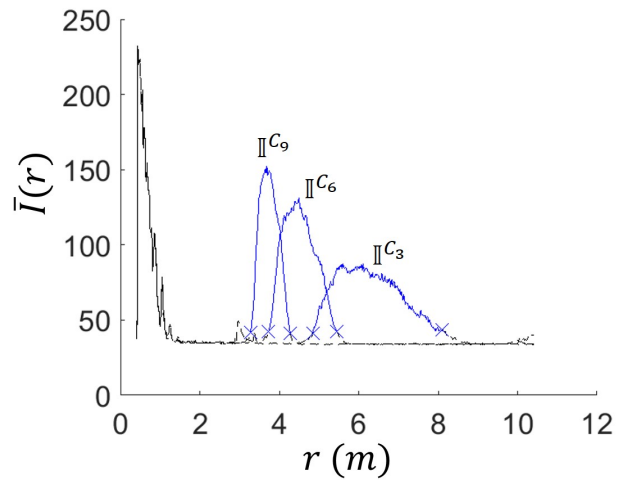

Fig. 21. Extraction of background region of beam profile based on threshold value.

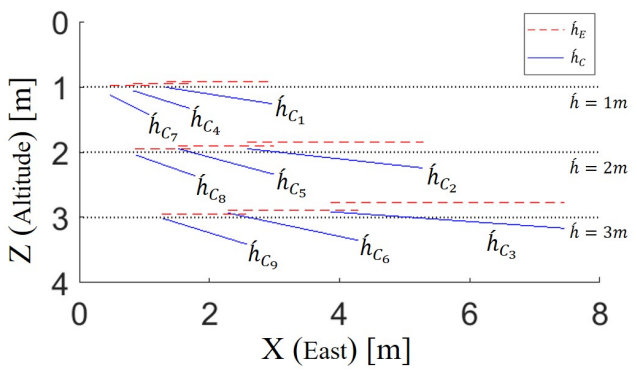

Fig. 22. Comparison result between proposed method and fixed vertical beam width based FSE estimation method.

defined as the fixed vertical beam width based FSE estimation method, and the results are compared for the performance verification of the proposed method. The comparison method used the sonar image of each condition as shown in Fig. 17, and estimates the FSE as follows. The boundary region $r_{\text {emin }}$ and $r_{\text {emax }}$ are selected based on the threshold value in the beam profile $\mathbb{I}^{C_{n}}$ at the condition $M_{n}$, in Fig. 21 . The leading and trailing edges of the background extracted in this way, are marked with white lines in the sonar image in Fig. 17.

The estimation results of the comparison method for the sonar image of each condition are shown in Fig. 22. In the result graph, the black dotted lines represent the actual altitude from the bottom of the water tank, and the red dash lines represent the FSE estimated by the proposed method. The blue solid lines show the FSE estimated by the comparison method. This line is represented by the line $\hat{h}_{C_{n}}$ connecting the estimated $h_{\text {emin }}$ and $h_{\text {emax }}$ based on the two edges at each condition $M_{n}$. All estimate results, $\hat{h}_{C_{n}}$ have a slope, and the slope is more gentle at the small tilt angle. The estimate result, $h_{C_{3}}$ shows more accurate result than our proposed method. However, we can confirm that there are larger errors in the results of comparison method. The main reason for this difference is that the vertical beam width is not always constant. Figure 12 shows that when the FSS scans a flat bottom surface, the effective vertical beam width decreases with increasing altitude. Especially, when the altitude is small, the effective angle change amount is large. The effective vertical beam width of the FSS is not a constant value. The FSE results estimated with these assumptions are not valid 


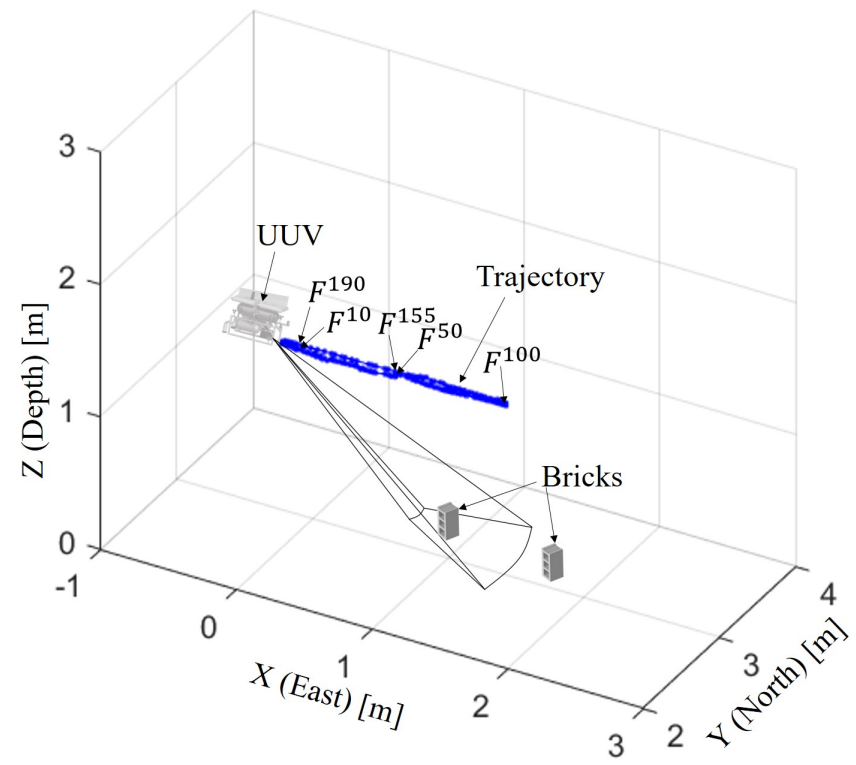

Fig. 23. Experimental setting to verify the robustness of the proposed method if there are objects on the floor.

because of the large error.

\section{E. Experiment for robustness of proposed method}

If there is no object on the seafloor as in the above experiment, the results of FSE estimation are correct. Additional experiments were conducted to verify the robust performance of proposed method when there is an object on the floor. In the same condition as in the previous experiment, two bricks were placed on the floor as shown in Fig. 23, and the UUV moved back and forth to scan the bottom and estimate the FSE. The size of each brick is $200 \times 150 \times 300 \mathrm{~mm}$, and the two bricks are spaced about $600 \mathrm{~mm}$ apart. Figure 24 shows the estimation result of the FSE of the UUV moving over the bricks. Experimental results show that the FSE, $h_{E}$ is well estimated in the most of the sections, except for some sections with errors. The largest error is about $100 \mathrm{~mm}$ near the frame 50 (F50) and the frame 150 (F150). Figure 25 (b) and (e) show the sonar image at F50 and F150, and the crosstalk phenomenon occurs near the highlight area of the object where in front of the effective region. This phenomenon causes that the highlights of the object blur into the surrounding, and effects extending the boundary $r_{\text {emin }}$ of the effective region to the highlight area of the object. As a result, the beam distribution pattern is abnormally deformed, causing an error in matching with the template.

Using the sonar image that removed the crosstalk area which causes the error would be much better. There have been several studies on removal or mitigation of crosstalk in FSS images [9], [22], [23], [24]. Among the various removal methods, we used convolutional-neural-network-based crosstalk removal method proposed by Sung et al. [25], and the results are shown in Fig. 25 (c) and (f). The removal processed frames, $F_{c}^{50}$ and $F_{c}^{155}$ show that the crosstalk around the highlights of the object is very well removed. This removal effect can be better seen

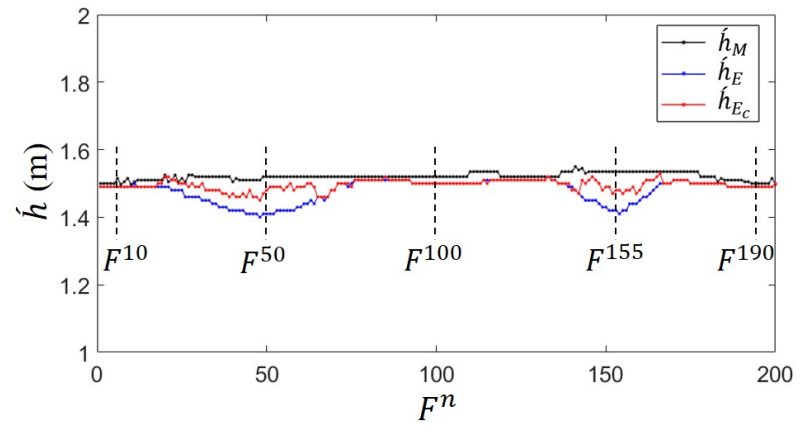

Fig. 24. Results of estimated FSE of robust experiment with obstacles.

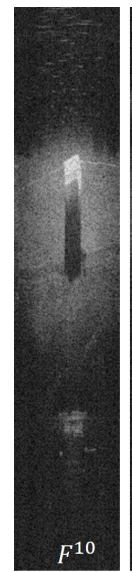

(a)

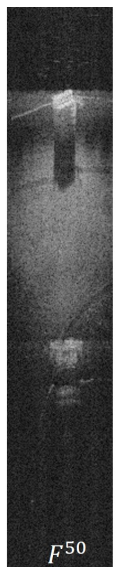

(b)

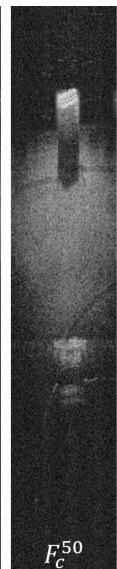

(c)

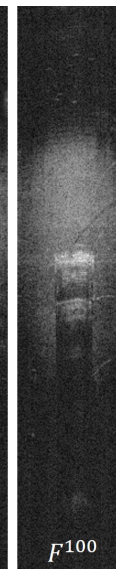

(d)

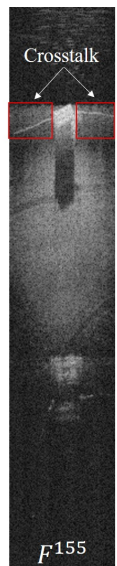

(e)

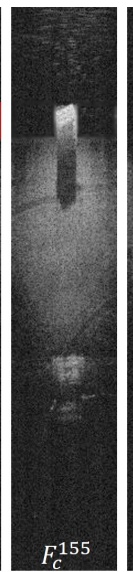

(f)

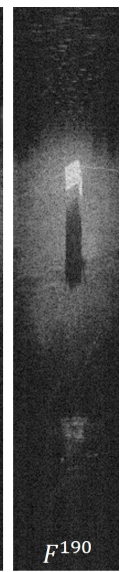

(g)
Fig. 25. Frames acquired during robustness experiments; (a), (b), (d), (e) and (g) are raw frames, (c) and (f) are frames which the crosstalk effect has been removed.

by at the beam pattern in Fig. 26. In Fig. 26 (c), the beam pattern, $\mathbb{I}^{M}$ becomes the beam pattern, $\mathbb{I}^{M_{c}}$, when the front area with high intensity value due to crosstalk is removed. The matched template pattern, $\mathbb{I}^{E_{c}}$ of the frame with crosstalk removed shows a result of shifting closer to the actual value as compared with the previously matched template pattern, $\mathbb{I}^{E}$. It can be seen from Fig. 24 that the results of the estimated FSE are much improved due to the change in matched template pattern.

\section{CONCLUSION}

The proposed method is based on modeling the propagation process of acoustic beams from the transmitter to the receiver while considering the environment altogether. The parameters of the proposed model, which take into account the environmental factors, can be updated each time via the DA experiment before the FE experiment. The proposed method is a practical solution to estimate FSE compared to the existing methods. We performed indoor experiments to verify the accuracy of the proposed method. These experiments were performed to compare the estimation results of the proposed method with measured results using the UUVs in an indoor water tank, and our experimental results show that the errors of the proposed method were less than $10 \%$. Moreover, the 


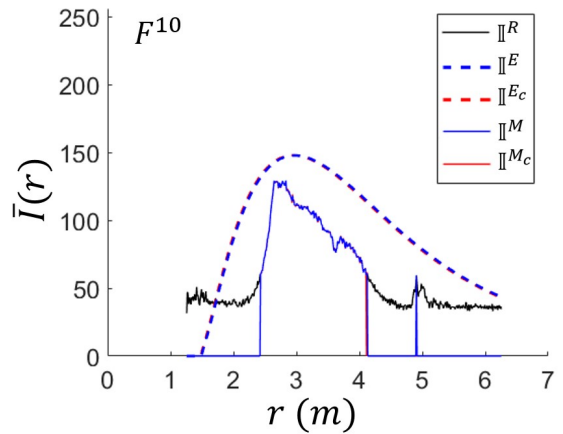

(a)

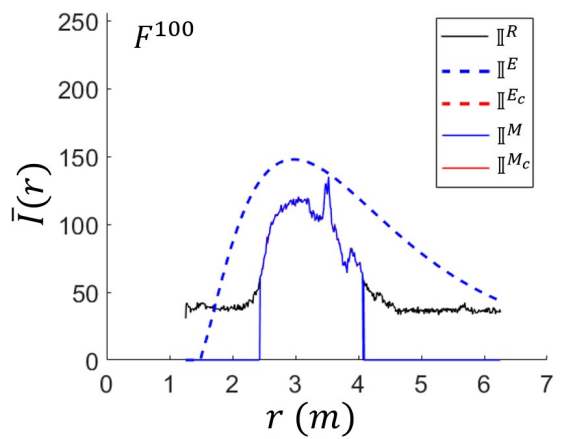

(b)

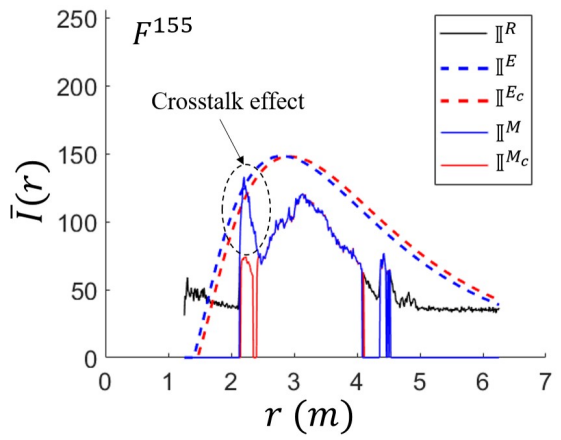

(c)

Fig. 26. Beam profiles and best matched templates of each frame, (a) Fame 10, (b) Frame 100, (c) Frame 155.

proposed method is verified to estimate the FSE well even if there are objects on the seafloor by the robustness experiment. In the next studies, we plan to verify FSL estimation accuracy of the proposed method for various background environment of field, such as a sand, mud, or gravel. Experiments on these various seabed conditions can be used to database model parameters for various environments. If the model parameters at the seabed are accumulated in such a database, the FE experiment can be performed without performing DA experiment every time. Furthermore, in order to overcome the problem that the error of the estimated FSE is caused in lower tilt angle of FSS, it is necessary to add a parameter term for the beam angle to the FSS model.

\section{ACKNOWLEDGMENT}

This research was supported by the National Research Foundation of Korea(NRF) grant funded by the Korea gov-
ernment(MSIT) (No. 2017R1A5A1014883).

\section{REFERENCES}

[1] J. Yuh, "Design and control of autonomous underwater robots: A survey," Autonomous Robots, vol. 8, no. 1, pp. 7-24, 2000.

[2] L. L. Whitcomb, "Underwater robotics: Out of the research laboratory and into the field," in Robotics and Automation, 2000. Proceedings. ICRA'O0. IEEE International Conference on, vol. 1. IEEE, 2000, pp. 709-716.

[3] H. Kondo, S. Yu, and T. Ura, "Object observation in detail by the auv" tri-dog 1" with laser pointers," in OCEANS, 2001. MTS/IEEE Conference and Exhibition, vol. 1. IEEE, 2001, pp. 390-396.

[4] T. Kim and J. Yuh, "Development of a real-time control architecture for a semi-autonomous underwater vehicle for intervention missions," Control Engineering Practice, vol. 12, no. 12, pp. 1521-1530, 2004.

[5] A. Palomer, P. Ridao, and D. Ribas, "Multibeam 3d underwater slam with probabilistic registration," Sensors, vol. 16, no. 4, p. 560, 2016.

[6] B. He, Y. Liang, X. Feng, R. Nian, T. Yan, M. Li, and S. Zhang, "Auv slam and experiments using a mechanical scanning forward-looking sonar," Sensors, vol. 12, no. 7, pp. 9386-9410, 2012.

[7] M. D. Aykin and S. Negahdaripour, "On feature matching and image registration for two-dimensional forward-scan sonar imaging," Journal of Field Robotics, vol. 30, no. 4, pp. 602-623, 2013.

[8] - "Forward look 2d sonar image formation and 3d reconstruction," in Oceans-San Diego, 2013. IEEE, 2013, pp. 1-10.

[9] E. Belcher, W. Hanot, and J. Burch, "Dual-frequency identification sonar (didson)," in Underwater Technology, 2002. Proceedings of the 2002 International Symposium on. IEEE, 2002, pp. 187-192.

[10] H. Cho, B. Kim, and S.-C. Yu, "Auv-based underwater 3-d point cloud generation using acoustic lens-based multibeam sonar," IEEE Journal of Oceanic Engineering, 2017.

[11] N. Hurtós, "Forward-looking sonar mosaicing for underwater environments," Ph.D. dissertation, Ph. D. dissertation, Universitat de Girona, 2014.

[12] M. Grasmueck, G. P. Eberli, D. A. Viggiano, T. Correa, G. Rathwell, and J. Luo, "Autonomous underwater vehicle (auv) mapping reveals coral mound distribution, morphology, and oceanography in deep water of the straits of florida," Geophysical Research Letters, vol. 33, no. 23, 2006.

[13] J. Pyo, H.-G. Joe, J.-H. Kim, A. Elibol, and S.-C. Yu, "Development of hovering-type auv cyclops for precision observation," in Oceans-San Diego, 2013. IEEE, 2013, pp. 1-5.

[14] J.-H. Gu, H.-G. Joe, and S.-C. Yu, "Development of image sonar simulator for underwater object recognition," in Oceans-San Diego, 2013. IEEE, 2013, pp. 1-6.

[15] H. Cho, J. Pyo, J. Gu, H. Jeo, and S.-C. Yu, "Sonar simulator-based underwater object recognition and its tolerance evaluation," in OCEANS 2016-Shanghai. IEEE, 2016, pp. 1-7.

[16] J. Pyo, H. Cho, and S.-C. Yu, "Beam slice-based recognition method for acoustic landmark with multi-beam forward looking sonar," IEEE Sensors Journal, vol. 17, no. 21, pp. 7074-7085, 2017.

[17] Q. Li, Digital sonar design in underwater acoustics: principles and applications. Springer Science \& Business Media, 2012.

[18] R. J. Urick, Principles of underwater sound for engineers. Tata McGraw-Hill Education, 1967.

[19] A. Papoulis and S. U. Pillai, Probability, random variables, and stochas tic processes. Tata McGraw-Hill Education, 2002.

[20] F. B. Jensen, W. A. Kuperman, M. B. Porter, and H. Schmidt, Computational ocean acoustics. Springer Science \& Business Media, 2000.

[21] S. Negaharipour, "On 3-d scene interpretation from fs sonar imagery," in 2012 Oceans. IEEE, 2012, pp. 1-9.

[22] K.-W. Jorg and M. Berg, "First results in eliminating crosstalk and noise by applying pseudo-random sequences to mobile robot sonar sensing," in Intelligent Robots and Systems' 96, IROS 96, Proceedings of the 1996 IEEE/RSJ International Conference on, vol. 1. IEEE, 1996, pp. 292 297.

[23] S. Shoval and J. Borenstein, "Using coded signals to benefit from ultrasonic sensor crosstalk in mobile robot obstacle avoidance," in Robotics and Automation, 2001. Proceedings 2001 ICRA. IEEE International Conference on, vol. 3. IEEE, 2001, pp. 2879-2884.

[24] A. Asada, H. Kunishima, T. Igarashi, T. Nagase, T. Matsuda, and K. Shibata, "Advanced mosaic techniques of acoustic video images for underwater surveillance and diagnosing degradation levels of harbor structures," in Proceedings of Underwater Acoustic Measurements: Technologies and Results, vol. 1. Citeseer, 2009, pp. 227-234. 
This article has been accepted for publication in a future issue of this journal, but has not been fully edited. Content may change prior to final publication. Citation information: DOI 10.1109/JSEN.2019.2918512, IEEE Sensors

[25] M. Sung, H. Cho, H. Joe, B. Kim, and S.-C. Yu, "Crosstalk noise detection and removal in multi-beam sonar images using convolutional neural network," in OCEANS 2018 MTS/IEEE Charleston. IEEE, 2018, pp. 1-6. 